\title{
La longue et sinueuse route vers l'emploi : les carrières d'insertion professionnelle des réfugiés sur le marché du travail en Belgique
}

The Long and Winding Road to Employment: Labour Market Careers of Refugees in Belgium

La larga y tortuosa vía hasta el empleo: carreras de inserción profesional de refugiados en el mercado de trabajo en Bélgica

\section{Barbara Herman et Andrea Rea}

\section{(2) OpenEdition}

\section{Journals}

Édition électronique

URL : https://journals.openedition.org/remi/9467

DOI : $10.4000 /$ remi.9467

ISSN : $1777-5418$

Éditeur

Université de Poitiers

Édition imprimée

Date de publication : 1 décembre 2017

Pagination : 109-134

ISBN : 979-10-90426-60-3

ISSN : 0765-0752

Référence électronique

Barbara Herman et Andrea Rea, « La lonque et sinueuse route vers l'emploi : les carrières d'insertion professionnelle des réfugiés sur le marché du travail en Belgique », Revue européenne des migrations internationales [En ligne], vol. 33 - n4 | 2017, mis en ligne le 01 décembre 2019, consulté le 14 avril 2022. URL : http://journals.openedition.org/remi/9467 ; DOI : https://doi.org/10.4000/remi.9467 


\title{
La longue et sinueuse route vers I'emploi : les carrières d'insertion professionnelle des réfugiés sur le marché du travail en Belgique
}

\section{Barbara Herman ${ }^{1}$ et Andrea Rea ${ }^{2}$}

\begin{abstract}
Bien que l'accroissement du nombre d'étrangers résidant en Europe tienne notamment à l'arrivée de nombreux demandeurs d'asile et réfugiés, peu d'études leur sont spécifiquement consacrées. Au cours des trente dernières années, on dénombre plus de travaux consacrés au travail des sans-papiers qu'à celui des demandeurs d'asile et des réfugiés, sauf au Canada. II en va de même en Belgique. Certains travaux (Martiniello et al., 2010 ; Mussche et al., 2010 ; Timmerman et al., 2014) se penchent sur la question de l'intégration de l'ensemble des nouveaux migrants sur le marché du travail sans s'intéresser spécifiquement à la situation des demandeurs d'asile et des réfugiés. D'autres recherches ont, par contre, mis l'accent sur l'intégration sur le marché du travail des migrants en situation irrégulière ou régularisés (Wets, 2009 ; Marx et al., 2008). Les résultats présentés dans cette contribution sont issus d'une recherche originale portant spécifiquement sur l'intégration sur le marché du travail des demandeurs d'asile et des réfugiés en Belgique et tenant compte des principales interprétations fournies par la littérature scientifique. Nous montrerons en quoi le temps joue un rôle central dans le processus d'intégration économique.
\end{abstract}

Les recherches consacrées à l'intégration sur le marché du travail des demandeurs d'asile et des réfugiés reconnus montrent que leurs taux d'emploi sont relativement bas (OCDE, 2008). Un rapport récent de I'OCDE (2016) relève que les taux d'emploi des réfugiés sont plus bas que ceux des nationaux, des immigrés de deuxième génération et des autres immigrés arrivés avec un autre statut (Piché et al., 2002 ; Bevelander, 2011). En Suède, le taux d'emploi des personnes arrivées dans le cadre du regroupement familial est plus élevé que celui des réfugiés (Bevelander, 2011).

\footnotetext{
1 Chercheuse en sociologie, Université Libre de Bruxelles (ULB), Groupe de recherche sur les Relations Ethniques, les Migrations et I'Égalité (GERME), avenue Jeanne 44, CP124, B-1050 Bruxelles, Belgique; bherman@ulb.ac.be

2 Professeur de sociologie, Université Libre, de Bruxelles (ULB), Groupe de recherche sur les Relations Ethniques, les Migrations et I'Égalité (GERME), avenue Jeanne 44, CP124, B-1050 Bruxelles, Belgique; area@ulb.ac.be
} 
Dans la littérature, les facteurs expliquant l'intégration sur le marché du travail sont généralement de trois ordres : les facteurs individuels (âge, genre, état civil, nationalité, etc.), les facteurs incitant à l'accès à I'emploi (longueur du séjour, statut du séjour, connaissance linguistique, niveau d'étude, capital social, etc.) et les caractéristiques structurelles et institutionnelles (opportunités du marché du travail, barrières à l'emploi, taux de chômage local, législation relative aux demandeurs d'asile, politiques d'intégration, etc.). Wooden (1991) montre qu'en Australie les taux d'emploi des réfugiés sont faibles au moment de l'arrivée, mais qu'ils croissent avec la longueur du séjour. Le temps est aussi un des facteurs déterminants pour Piguet et Wimmer (2000) parce qu'il permet à la fois une plus grande maîtrise linguistique et une meilleure connaissance institutionnelle favorisant un accroissement de la mobilisation des réseaux sociaux. À partir de données récentes, le rapport de l'OCDE (2016) arrive à la même conclusion.

De nombreuses recherches montrent que les femmes ont moins de probabilité d'avoir un emploi que les hommes (Piguet et Wimmer, 2000 ; Piché et al., 2002 ; Bevelander, 2011 ; Bloch, 2007). De même, avoir un enfant accroît la probabilité d'avoir un emploi pour les hommes, mais pas pour les femmes. Les femmes ont plus de probabilité d'avoir un emploi si elles sont célibataires. L'intégration sur le marché du travail est liée à l'âge et au niveau d'études : les plus jeunes et les plus diplômés sont le plus souvent ceux qui ont un emploi. Cependant, les réfugiés sont aussi confrontés à leur surqualification et aux difficultés à faire reconnaître leurs diplômes du pays d'origine (Bloch, 2007). Les réfugiés hautement qualifiés sont confrontés à des processus de déqualification sur le marché du travail (Krahn et al., 2000 ; Lamba, 2003). Pour certains réfugiés, la migration s'apparente alors à une expérience de déclassement social. Par ailleurs, la connaissance de la langue joue aussi un rôle très important dans l'insertion sur le marché du travail. Ainsi, la corrélation positive entre connaissance linguistique du pays d'installation et insertion sur le marché du travail a été établie dans divers travaux (Krahn et al., 2000 ; Colic-Peisker etTilbury, 2006).

Des études insistent sur l'importance de l'origine nationale, mais la situation peut être contrastée. Ainsi aux États-Unis, les Vietnamiens ont dans certaines études des taux d'occupation élevés et dans d'autres très bas (Montgomery, 1991 ; Tran, 1991). Piché et al. (2002) montrent qu'au Québec, l'origine nationale joue un rôle important lorsque les variables socioéconomiques et le niveau de formation sont contrôlés. Les personnes provenant des pays en voie de développement rencontrent plus de difficulté à accéder à un premier emploi que celles provenant des pays d'Europe de l'Est. Au Royaume-Uni, les personnes provenant de pays anglophones ont plus de probabilité d'être actives sur le marché du travail (Bloch, 2007). Hjarno (1991) estime que la faiblesse des réseaux sociaux et les stéréotypes expliquent les difficultés rencontrées par les réfugiés à intégrer le marché du travail alors que Lamba (2003) considère que les réseaux sociaux mobilisés par les réfugiés ne sont pas suffisants pour compenser leur plus faible mobilité dans I'emploi. Enfin, dans un autre registre, Hauff et Vaglum (1993) insistent sur un facteur spécifique pour expliquer leur difficulté à intégrer le marché de l'emploi : les impacts des traumatismes vécus par les réfugiés et les violences qu'ils ont subies. 
L'emploi des réfugiés est très sensible à la conjoncture locale. Là où le taux de chômage est élevé, les taux d'occupation sont faibles. De même, les opportunités d'emploi sont plus nombreuses dans les grandes villes où le secteur des services est plus important (Belevander, 2011). En outre, certains réfugiés reconnus reçoivent des prestations sociales qui leur permettent de renoncer à des emplois mal payés et peu valorisés, mais ceci varie selon les régimes nationaux d'État social, comme le suggère la comparaison entre les Pays-Bas et I'Italie (Korac, 2003). Au Canada où les études longitudinales sur l'insertion professionnelle (Yu et al., 2007) sont fréquentes, Renaud et al. (2003) ont montré que les réfugiés sélectionnés à l'étranger (les réinstallés) ont des taux d'emploi plus élevés que ceux qui demandent l'asile sur place. La cause de cette différence ne réside pas dans les caractéristiques des deux groupes, mais dans le parcours institutionnel plus complexe auquel sont confrontés les réfugiés ayant demandé l'asile sur place.

Plusieurs travaux mettent aussi en évidence un paradoxe : le taux d'emploi des demandeurs d'asile est parfois plus élevé que celui des réfugiés. Ceci tient à la plus grande disposition des demandeurs d'asile à accepter des emplois sous-qualifiés dans les secteurs traditionnels des nouveaux migrants (construction, restauration et hôtellerie) (Piguet et Wimmer, 2000). Dans l'agriculture, les demandeurs d'asile ont parfois remplacé les saisonniers. Lorsqu'ils obtiennent le statut de réfugié, les personnes changent de secteur d'activité et cherchent un emploi dans un secteur plus en lien avec leur qualification. Cette même observation a été établie en Belgique pour les sans-papiers ayant obtenu une régularisation de leur statut (Marx et al., 2008).

Cet article s'articule en trois parties. La première partie présente les données administratives utilisées pour analyser les carrières d'insertion socioéconomique ainsi que la définition de la population et la période sur laquelle nous avons travaillé. Dans cette partie, l'ensemble des carrières socioéconomiques observées au sein de la population sélectionnée est également présenté. La deuxième partie présente les résultats de l'analyse séquentielle permettant de construire des typologies d'insertion professionnelle. Cette partie met en évidence le rôle central du temps dans le processus d'intégration économique et évalue l'impact des différents facteurs individuels et contextuels sur les trajectoires d'intégration sur le marché du travail par le biais de régressions logistiques multivariées. Enfin, la troisième partie présente une analyse de l'évolution des secteurs d'activité pour les réfugiés qui occupaient un emploi.

\section{Une enquête longitudinale des carrières d'insertion}

La recherche que nous avons menée (Rea et Wets, 2015) vise, d'une part, à analyser l'intégration sur le marché du travail de tous les demandeurs d'asile, réfugiés et personnes s'étant vu accorder un statut de protection subsidiaire entre janvier 2001 et décembre 2010, et d'autre part, à créer une typologie des parcours d'intégration. À partir du concept de carrière migratoire (Martiniello et Rea, 2014), nous avons cherché à élucider la manière dont les conditions structurelles, institutionnelles et politiques, les caractéristiques individuelles (âge, genre, situation familiale, nationalité, etc.) et la mobilisation des réseaux sociaux déterminent les carrières d'insertion professionnelle. 
La recherche repose sur I'utilisation des bases de données administratives existantes en Belgique relative au statut de séjour (le Registre national) et à la situation en regard de la sécurité sociale et de l'emploi (Banque carrefour de la sécurité sociale). Le Registre national (RN) constitue un registre centralisé de la population placé sous la responsabilité du ministère de I'Intérieur. II comporte plusieurs sous-registres. Dans le registre de la population figurent les réfugiés reconnus, alors que les demandeurs d'asile sont encodés dans le registre d'attente. Ces registres consignent des informations telles que le nom, l'âge, le genre, la nationalité, I'historique de nationalité, l'historique de lieu de résidence et l'évolution du statut de séjour. Le Registre national dispose ainsi de toutes les informations relatives aux étapes de la procédure de la demande d'asile. Placée sous la responsabilité du ministère des Affaires sociales, la Banque carrefour de la sécurité sociale (BCSS) renferme des informations relatives au marché du travail (statut, employeur, cotisations de sécurité sociale, temps de travail, chômage, aide sociale, etc.), des informations relatives aux retraites, à la maladie/invalidité/ incapacité de travail et aux allocations familiales. Ces données sont enregistrées tous les trimestres pour toutes les personnes résidant en Belgique. Celles recueillies pour notre recherche résultent du couplage de ces deux bases de données. Cependant, ces bases de données administratives présentent aussi des limites en regard du cadre théorique mobilisé des carrières migratoires (Martiniello et Rea, 2014). Deux données importantes sont absentes : d'une part, le niveau d'étude ou de formation et d'autre part, la mobilisation des réseaux sociaux.

Nous disposons des données relatives à tous les demandeurs d'asile ayant

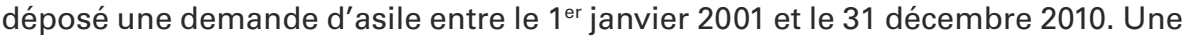
base de données incluant des informations relatives à 108856 individus a ainsi été constituée. Les données utilisées ne constituent pas un échantillon, mais représentent toutes les personnes qui ont eu le statut de demandeurs d'asile durant la période retenue. Nous avons effectué une première analyse descriptive sur les personnes de plus de dix-huit ans et de moins de soixante-cinq ans, soit 71768 personnes (Rea et Wets, 2015). L'analyse de ces données offre uniquement un point de vue statique puisqu'elle présente la situation à un moment donné. Cette analyse descriptive est complétée d'un examen de l'insertion professionnelle à partir d'une approche longitudinale qui fournit une image dynamique des changements de statuts individuels sur le marché de l'emploi. Ceci nous a offert la possibilité de construire des carrières d'emploi.

Cette contribution ne porte que sur l'analyse longitudinale de l'insertion professionnelle sur quatre ans de toutes les personnes ayant obtenu le statut de réfugié en 2003, 2004, 2005 et 2006, soit 4869 personnes. Nous avons choisi ces années afin de neutraliser les effets potentiels de deux changements législatifs majeurs : I'attribution d'un permis de travail à tous les demandeurs d'asile après six mois de procédure en 2003 et le changement dans la procédure d'examen des demandes d'asile en $2007^{3}$. Les carrières des réfugiés étudiés n'ont pas été affectées par le changement de 2007 parce qu'il n'y avait pas d'effet rétroactif

3 Le changement de la loi de 2003 consiste à octroyer automatiquement le permis de travail $\mathrm{C}$ à tous les demandeurs d'asile qu'ils l'utilisent ou non. Précédemment, les demandeurs $d^{\prime}$ asile devaient demander le permis de travail B s'ils voulaient travailler. La loi de 2003 vient étendre une situation préexistante puisque les demandeurs d'asile peuvent déjà travailler, mais ils n'ont plus besoin d'entamer une procédure de demande de permis de travail. 
de cette loi. Au cours de ces quatre années, l'économie belge a bien résisté à la crise économique et financière mondiale comme l'indique le rapport de la Banque nationale de Belgique (2011). La croissance était de 1,9\% et l'emploi s'est accru durant la même période. Les réfugiés proviennent essentiellement d'Europe de l'Est (ex-Yougoslavie), de Russie (de nombreux Tchétchènes) et d'Afrique centrale. L'obtention du statut de réfugié s'accompagne de l'accroissement de droits : permis de résidence permanente, droit à une aide sociale mensuelle $(+/-850 €)$, accès au logement social, à la formation professionnelle et à la nationalité après cinq ans.

Une carrière se définit comme une succession de différentes positions sur le marché du travail. Les données de la Banque carrefour de la sécurité sociale permettent de déterminer aisément la position socioéconomique d'un individu le dernier jour de chaque trimestre. Nous avons enregistré le statut social (de travail ou de non-travail) de tous les réfugiés durant quatre ans (seize trimestres) en fusionnant les quatre séries temporelles et en prenant comme même point de départ le trimestre qui suit celui de l'obtention du statut de réfugié. Afin d'analyser les carrières, cinq catégories de statut sur le marché du travail ont été identifiées : (1) salarié, (2) indépendant, (3) chômeur bénéficiant d'allocations de chômage, (4) aide sociale et (5) " autre $"^{4}$. Nous avons limité la subdivision de la position socioéconomique à ces cinq catégories pour des raisons d'ordre méthodologique : dans l'analyse séquentielle, plus il y a de catégories, plus le nombre de carrières pouvant être distinguées les unes des autres est élevé et plus la probabilité de parvenir à une typologie des carrières significative est faible (Robette, 2011).

Avant de présenter la typologie des carrières, regardons la situation de ces 4869 personnes sur le marché de l'emploi au cours des seize trimestres. La figure 1 montre la répartition des statuts des réfugiés pour chaque trimestre. II ne s'agit pas ici de données longitudinales. II apparait qu'au moment de la reconnaissance de leur statut, $56,7 \%$ des réfugiés bénéficiaient de l'aide sociale. Cette proportion décroît jusqu'à la fin de la période étudiée (24,9\%). Nous définissons une personne active sur le marché du travail selon la définition proposée par le Bureau international du travail à savoir une personne qui est, soit occupée (employée ou indépendante), soit demandeuse d'emploi indemnisée. Tout au long des quatre années, la proportion d'actifs (salarié + demandeur d'emploi + indépendant) devient plus importante en passant de $18,9 \%$ au moment de la reconnaissance du statut de réfugié à $56,9 \%$ en fin de période. En fin de période, les personnes disposant d'un emploi sont $42 \%$, les chômeurs indemnisés 13,4 \% et les indépendants 1,5 \% (cf. Figure 2). Comme le relèvent de nombreuses études (Piguet et Wimmer, 2000 ; Wooden, 1991 ; Piché et al., 2002 ; OCDE, 2016), I'intégration sur le marché du travail s'améliore avec le temps et atteint un niveau relativement élevé. Les personnes classées dans la catégorie " autre " représentent, en fin de période, $18,2 \%$ et donc sont légèrement moins nombreuses qu'en début de période. Ce premier résultat met en exergue qu'avec le temps les réfugiés contribuent positivement à la vie économique du pays, comme l'a aussi mis en évidence un article de la Banque nationale de Belgique (Burggraeve et Piton, 2016).

4 Les personnes titulaires de ce statut n'ont aucun revenu légal et elles sont assimilées aux catégories de femmes ou d'hommes au foyer. 
Figure 1 : Distribution des statuts socioéconomiques par trimestre

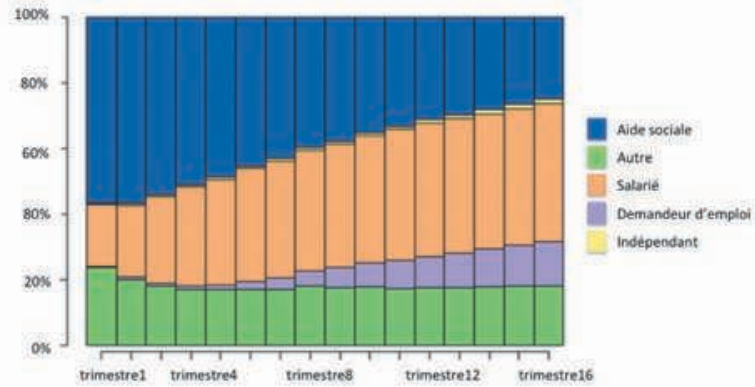

Sources : RN et BCSS (2003-2010); calcul des auteurs ${ }^{5}$.

Figure 2 : Proportion des statuts socioéconomiques au début et à la fin de la carrière
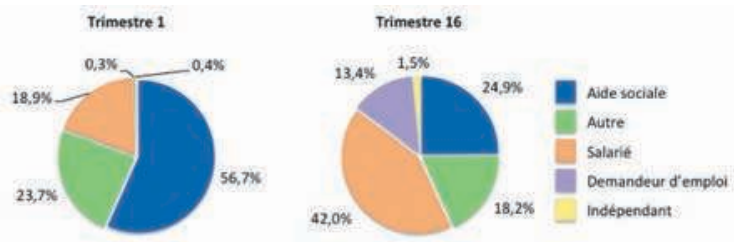

\section{La typologie des carrières d'insertion}

L'analyse longitudinale fait apparaître une grande variété des carrières d'insertion professionnelle. Sur les quatre ans, les dix carrières d'insertion professionnelle totalement identiques ne représentent que $25,2 \%$ de la population étudiée. La grande dispersion nous a ainsi conduits à produire des types idéaux de carrières. Cette étape offre la possibilité de répartir la population en un nombre limité de groupes relativement homogènes et distincts les uns des autres, en identifiant des carrières types. Dans une seconde étape, nous analysons les caractéristiques individuelles des réfugiés repris dans chacune des carrières types. Lors d'une première étape, nous avons réalisé une analyse séquentielle avec appariement optimal (optimal matching). Cette méthode statistique permet d'identifier les carrières proches de celles distantes. Ensuite, l'analyse par grappes (cluster analysis) permet d'obtenir des carrières idéaltypiques (Abbott et Tsay, 2000 ; Halpin et Chan, 1998 ; Robette, 2011). Cette méthode nous a conduit à différencier quatre carrières d'insertion professionnelle $^{6}:(1)$ la carrière active, (2) la carrière progressivement active, (3) la carrière de dépendance à I'aide sociale et (4) la carrière du statut " autre ». Toutes ces

5 Tous les calculs des figures et tableaux de l'article ont été faits par les auteurs et les données proviennent du Registre national (RN) et de la Banque carrefour de la sécurité sociale (BCSS) (2003-2010).

6 L'identification du nombre de grappes d'une typologie est liée à la fois à des critères statistiques, mais doit également être cohérente et porteuse d'enseignements du point de vue de la recherche menée (Robette, 2011). Par conséquent, avant de sélectionner cette typologie à quatre grappes, nous avons observé d'autres typologies à différents niveaux de partition afin d'examiner la nature et I'homogénéité de chaque classe par l'intermédiaire des sous-classes qui la composent. 
analyses ont été effectuées avec le logiciel R. Le tableau 1 montre la distribution des réfugiés en fonction de leur appartenance à ces quatre carrières ainsi que la proportion de femmes au sein de chacun de ces groupes.

Les résultats relatifs à la distribution genrée des carrières sont frappants. Alors que dans les deux premières carrières (active et progressivement active), seulement $34,5 \%$ et $35 \%$ des réfugiés sont des femmes, ces dernières sont très fortement surreprésentées dans la catégorie " autre " ainsi que légèrement surreprésentée dans la carrière de dépendance à l'aide sociale. Concernant la durée de la procédure, les personnes ayant une carrière active ont en moyenne une durée de procédure plus longue que ceux ayant un autre type de carrière. Étant donné que le moment de l'obtention du statut de réfugié définit le début de la carrière observée, la durée de la procédure équivaut au nombre d'années passées en Belgique depuis l'introduction de la demande d'asile. II semble qu'une carrière active sur le marché du travail regrouperait des réfugiés qui sont, en moyenne, en Belgique depuis plus longtemps que les autres types de carrières. Le temps constitue en cela un facteur d'intégration socioéconomique.

Tableau 1 : Séquences relatives aux carrières socioéconomiques selon l'appartenance à un cluster

\begin{tabular}{lcccc}
\hline Carrières & Fréquence & $\%$ & $\begin{array}{c}\text { Proportion de } \\
\text { femmes (en \%) }\end{array}$ & $\begin{array}{c}\text { Durée moyenne } \\
\text { de la procédure } \\
\text { d'asile } \\
\text { (en années) }\end{array}$ \\
\hline 1 - Active & 1656 & 34 & 34,5 & 2,3 \\
2 - Progressivement active & 1199 & 25 & 35 & 1,8 \\
3 - De dépendance sociale & 1432 & 29 & 55 & 1,8 \\
4 - " Autre " & 582 & 12 & 73,5 & 1,9 \\
Total & 4869 & 100 & 45 & 2 \\
\hline
\end{tabular}

L'étape suivante consiste à vérifier si cette typologie des carrières d'insertion professionnelle peut être attribuée à différentes caractéristiques individuelles ou contextuelles. Pour ce faire, la méthode statistique utilisée est la régression logistique multiple (DeMaris, 1992) ${ }^{7}$. Les variables indépendantes introduites dans notre modèle d'analyse sont résumées en annexe dans le tableau 2 et les fréquences des variables indépendantes par carrière et par genre dans le tableau 3 en annexe.

Des variables indépendantes reprenant la situation pendant la procédure d'asile sont également prises en compte. Premièrement, la durée de la procédure d'asile est mobilisée. Ensuite, une variable indiquant si le réfugié a été actif sur le marché du travail pendant la procédure d'asile est également mesurée. Nous avons aussi regardé si une personne a été active sur le marché du travail le trimestre qui précède celui de la reconnaissance de son statut de réfugié. Tenir compte du fait qu'un réfugié soit actif pendant sa procédure d'asile est nécessaire pour comprendre la transition professionnelle après l'obtention

\footnotetext{
7 Dans une régression multiple, on peut examiner l'effet d'une série de variables indépendantes présentées simultanément (par exemple, la durée de la procédure d'asile sur une variable dépendante telle que le fait d'avoir une carrière active) en neutralisant l'effet des autres variables indépendantes. Pour ces analyses, nous avons réalisé des régressions logistiques dues au caractère binaire des variables dépendantes.
} 
du statut et son impact sur la situation ultérieure. De la sorte, il s'agit aussi de voir s'il existe une continuité sur le marché du travail avant et après l'acquisition du statut de réfugié.

Finalement, la variable contextuelle est mesurée uniquement par la Région (Flandre, Wallonie, Bruxelles) de la première inscription dans une administration communale en Belgique. Les bases de données utilisées ne proposent pas d'autres variables contextuelles. Dans un État fédéral comme la Belgique, les trois Régions recouvrent au moins deux dimensions ayant un impact sur l'intégration sur le marché du travail : la situation économique et les politiques publiques destinées aux étrangers. Dans notre analyse, nous ne pouvons pas évaluer l'impact distinct des deux composantes de cette variable contextuelle sur les carrières des réfugiés. En Flandre, d'une part, la situation économique est meilleure et donc les opportunités d'emploi plus fréquentes et d'autre part, il existe une politique interventionniste envers de nouveaux migrants, dont les réfugiés, qui sont aussi soumis à l'obligation de suivre un parcours d'intégration (cours de néerlandais, histoire de la Belgique, citoyenneté). En Wallonie, la situation économique est plus défavorable et le chômage plus élevé. Par ailleurs, il n'existait pas à l'époque de suivi obligatoire de l'intégration des réfugiés. Toutefois, ils peuvent trouver des associations qui les soutiennent dans les démarches d'intégration. Cette même configuration se rencontre à Bruxelles où la situation de l'emploi est à mi-chemin entre la situation flamande et wallonne.

\section{La carrière active}

La figure 3 décrit le premier idéal-type. Pour chaque carrière, deux graphiques sont présentés. Le premier présente la distribution de chaque statut socioéconomique par trimestre et le deuxième représente l'ensemble des 1656 trajectoires individuelles de cette carrière. Cette carrière reprend $34 \%$ de la population des réfugiés et est la plus importante des quatre. Les personnes qui relèvent de cet idéal-type ont eu une carrière presque complètement active (employé, demandeur d'emploi indemnisé ou indépendant) durant toute la période. En moyenne, elles restent actives durant $79 \%$ de leur parcours. Le recours à I'aide sociale est donc quasi absent de leur carrière.

$D^{\prime}$ autre part, ce profil est caractérisé également par une augmentation de la proportion des demandeurs d'emploi indemnisés au fil du temps. Bien que nous considérions le statut de demandeur d'emploi indemnisé comme un statut actif sur le marché de l'emploi (étant donné que pour être chômeur rémunéré, il faut avoir déjà travaillé), nous observons qu'une minorité des personnes qui ont un emploi rémunéré ne le garde pas. En effet, en moyenne sur les quatre derniers trimestres, $12 \%$ des personnes qui ont ce type de carrière sont demandeurs d'emploi. 
Figure 3 : La carrière active

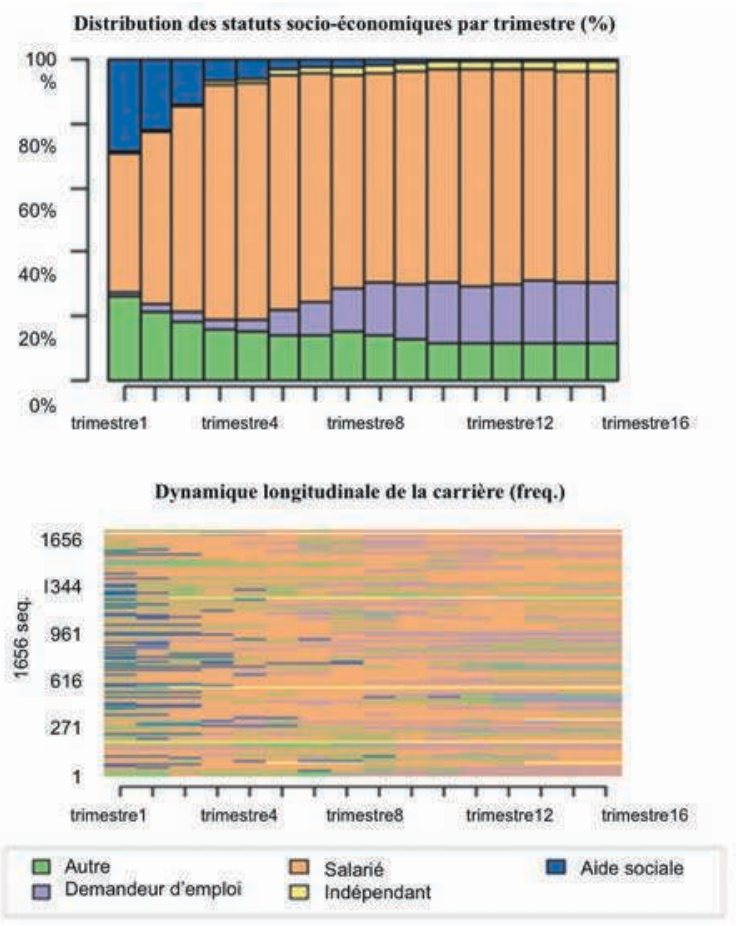

Afin de tenir compte de l'impact des caractéristiques individuelles, une régression logistique multiple a été appliquée en prenant comme variable dépendante, le fait d'avoir ou pas ce type de carrière (cf. Tableau 4 en annexe). Comme variables indépendantes, nous incluons toutes celles présentées dans le tableau 2 en annexe.

Concernant les caractéristiques individuelles, le genre a un impact sur le fait $d^{\prime}$ 'avoir ou pas une carrière active. La probabilité d'avoir ce type de carrière est supérieure pour les hommes. L'âge joue également un rôle. En particulier, les individus âgés de vingt-six à quarante ans ont une probabilité supérieure d'avoir une carrière active à ceux âgés de dix-huit à vingt-cinq ans et de quarante-et-un à soixante-et-un ans. Cela signifie que la relation entre l'âge et le fait d'avoir une carrière active est curvilinéaire : la probabilité croit jusqu'à un certain âge puis décroît jusqu'à l'âge de la retraite.

La situation familiale joue également un rôle et, en particulier, lorsque nous tenons compte de l'interaction entre la situation familiale et le genre. Les résultats en fonction du genre se trouvent en annexe dans le tableau 5 . Ces résultats montrent que la situation familiale des femmes influence fortement le fait d'avoir une carrière active sur le marché du travail alors que ce n'est pas le cas pour les hommes. En effet, les femmes ont une probabilité plus élevée d'être actives pendant toute la carrière observée si elles sont en couple, avec ou sans enfant, que si elles sont célibataires, et en particulier célibataires avec enfants. La situation homologue ne se produit pas pour les hommes étant donné qu'il n'y a quasiment aucun père célibataire dans notre population. 
L'origine nationale a un impact sur le fait d'avoir ce type de carrière. Comparées aux personnes originaires d'Europe de l'Est et du Caucase ainsi qu'aux personnes venant du Moyen-Orient, les personnes originaires d'Afrique subsaharienne ont une plus grande probabilité $d$ 'avoir une carrière active. Une interprétation probable, suivant ce qu'ont établi Colic-Peisker et Tilbury (2006), serait que les principaux pays de cette région (la République démocratique du Congo, la Côte d'Ivoire, le Burundi et la Guinée) sont des États où le français est une des langues officielles. La connaissance d'une des langues nationales belges avantagerait les réfugiés à s'insérer sur le marché du travail. La nationalité actuelle (avoir ou non la nationalité belge) n'a pas d'impact sur le fait d'avoir une carrière active. Ce sont d'autres variables telles que la situation nationale ou la nationalité d'origine qui ont plus d'impact sur le fait de développer ce type de carrière. En regardant la situation avant l'obtention du statut de réfugié, le nombre d'années de la procédure d'asile, qui équivaut au nombre d'années passées en Belgique, a un impact positif sur le fait d'avoir une carrière active : plus le nombre d'années est élevé, plus la probabilité d'avoir une carrière active est grande.

Par ailleurs, les personnes qui avaient déjà fait I'usage de la possibilité de travailler légalement avant l'obtention du statut ont plus de probabilité d'avoir ce type de carrière que celles qui n'ont pas travaillé pendant leur procédure d'asile. Comme observé précédemment (Marx et al., 2008), l'obtention du statut de réfugié vient consolider la situation des personnes qui étaient déjà bien insérées sur le marché du travail. Ce n'est pas la reconnaissance du statut de réfugié en soi qui initie l'insertion sur le marché de l'emploi. Cette reconnaissance du statut juridique vient plutôt renforcer la position de ces personnes sur le marché de l'emploi en enlevant les effets négatifs liés à l'insécurité du séjour. II importe particulièrement de relever l'effet positif que constitue l'accès au marché au travail durant la procédure d'asile (OCDE, 2016). Une plus forte intégration sur le marché du travail alors qu'ils/elles étaient demandeurs d'asile accroît la probabilité de maintenir une bonne insertion professionnelle.

Une analyse plus approfondie montre qu'il y a une interaction significative entre le fait de travailler pendant la procédure d'asile et la durée de la procédure. Plus une personne est en Belgique depuis longtemps, plus la probabilité de trouver un emploi déclaré pendant la procédure d'asile sera élevée, plus la probabilité d'avoir une carrière active sur le marché du travail belge après la reconnaissance du statut de réfugié sera élevée ${ }^{8}$.

Concernant l'impact des contextes institutionnels et économiques, il existe à nouveau une interaction avec le genre puisque ces contextes n'ont d'impact significatif que pour les femmes. Les femmes réfugiées qui ont habité en Région flamande et à Bruxelles au début de leur procédure d'asile ont significativement plus de probabilité d'avoir une carrière active pendant toute la période observée que celles habitant en Wallonie. Le contexte économique a donc une influence spécifique sur l'insertion active sur le marché du travail, notamment des femmes réfugiées. Sans avoir la possibilité d'évaluer précisément l'impact du contexte institutionnel qui en Flandre soutient de manière précoce l'intégration, on peut

8 En effet, les individus qui ont travaillé pendant la procédure d'asile et qui ont eu une procédure d'asile de plus de trois ans ont une probabilité quatre fois plus élevée d'avoir ce type de parcours que les individus qui n'ont pas travaillé et qui ont eu une procédure d'asile de moins d'un an. 
faire l'hypothèse que l'obligation du parcours d'intégration, et entre autres des cours de langue, joue aussi un rôle dans l'accès à l'emploi. Cette hypothèse suit les conclusions de l'étude portant sur l'intégration professionnelle des personnes ayant bénéficié de la campagne de régularisation en 2000 (Marx et al., 2008). Cependant, ces facteurs contextuels ne jouent pas pour les hommes, ce qui vient aussi tempérer le lien entre politique d'intégration interventionniste et insertion professionnelle. Ce résultat souligne surtout la situation plus délicate des femmes par rapport au contexte institutionnel et économique tout en mettant en évidence le rôle positif que peuvent jouer des politiques ciblées sur ce public.

\section{La carrière progressivement active}

Cette carrière qui concerne 1199 personnes est caractérisée par un parcours d'insertion active sur le marché de l'emploi après avoir obtenu le statut de réfugié. En effet, les personnes appartenant à cette catégorie ont débuté leur carrière essentiellement avec une aide sociale, et au fur et à mesure de leur trajectoire, la proportion de travailleurs augmente. Alors qu'au début de leur carrière, 49,7\% d'entre elles ont une aide sociale (cf. Figure 4) et $10 \%$ ont un emploi rémunéré, quatre années plus tard, la proportion d'aide sociale est seulement de $5 \%$ et de travailleurs actifs à $90 \%$. D'autre part, ce profil est caractérisé par une augmentation de la proportion des demandeurs d'emploi indemnisés. Ce cas de figure illustre très clairement une carrière avec une insertion progressive où l'obtention du statut de réfugié semble se révéler être un catalyseur en faveur de l'emploi. Cette carrière reprend $25 \%$ de la population.

Figure 4 : La carrière progressivement active
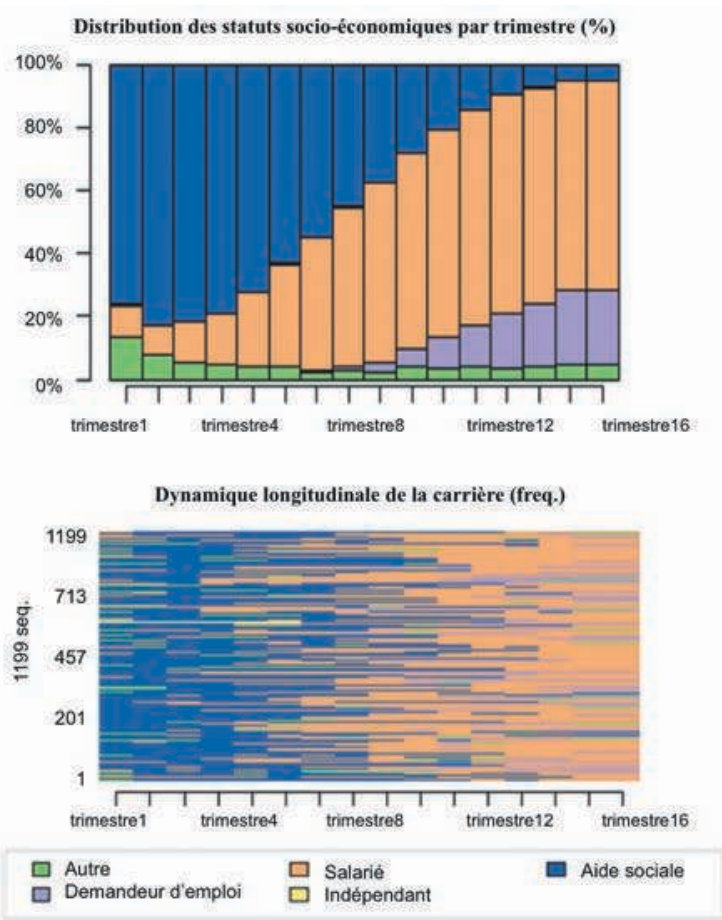
Le tableau 4 en annexe (colonne carrière 2) montre les résultats de la deuxième régression logistique multiple où la variable dépendante est le fait ou pas d'avoir une carrière progressivement active. Comme variables indépendantes, nous incluons toutes celles présentées dans le tableau 2 en annexe. Concernant le profil des personnes appartenant à ce type de carrière, les analyses en régression logistique montrent que les hommes ont plus de probabilité d'avoir ce type de carrière que les femmes. Par contre, l'âge n'est pas associé à une carrière d'insertion active progressive. II n'y a pas de différence significative entre les différentes catégories d'âges.

Encore une fois, la situation familiale joue un rôle important et, en particulier, lorsque l'interaction entre la situation familiale et le genre sont pris en compte (cf. Tableau 5 en annexe) : les femmes célibataires ont une plus grande probabilité d'avoir une carrière d'insertion progressive que les femmes en couple (avec ou sans enfant). Au sein du groupe des femmes célibataires, celles sans enfant ont plus de probabilité de se trouver dans cette carrière. En revanche, la situation familiale n'a aucun effet pour les hommes. En comparant ce résultat avec celui de la première carrière, où les femmes en couple (avec ou sans enfant) ont une plus grande probabilité d'avoir une carrière active que les femmes célibataires (avec ou sans enfant), nous pensons que ceci reflète la difficulté des femmes célibataires à trouver un emploi pendant la procédure d'asile. L'isolement peut constituer dans ce cas un handicap à l'insertion professionnelle. Une fois que le statut de réfugié a été reconnu, ces femmes auront plus de probabilité à s'insérer sur le marché du travail. Étant donné que ces résultats sont contrôlés par, notamment, la durée de la procédure d'asile, c'est-à-dire le nombre d'années passées en Belgique, nous pouvons faire l'hypothèse que la stabilisation du statut légal de ces femmes favoriserait une insertion active. Pour ces femmes, l'obtention du statut de réfugié serait un catalyseur de leur insertion professionnelle et de leur carrière. L'obtention du statut de réfugié leur permettrait d'acquérir ce qu'on pourrait nommer un capital juridique qui leur ouvrirait plus d'opportunités que lors de la situation antérieure. L'obtention du statut juridique en tant que capital juridique ouvre l'accès à des ressources sur deux dimensions. La première englobe les bénéfices liés au nouveau statut juridique de réfugié, notamment, l'accès au regroupement familial, au logement social, au permis de séjour illimité, à l'accès libre au marché du travail, etc. La deuxième dimension du capital juridique est liée à la possibilité de faire appel à des réseaux sociaux plus institutionnalisés et plus larges. Par l'acquisition du statut de réfugié, ces personnes et, en particulier les femmes, peuvent passer de liens sociaux centrés sur la famille ou les groupes de même origine ethnique basés sur des liens forts (Granovetter, 1973) vers des liens plus formels et des liens faibles donnant accès à de nouveaux réseaux sociaux plus diversifiés. En somme, l'obtention du statut de réfugié devient une condition pour ces femmes célibataires d'élargir et diversifier les réseaux sociaux afin d'accroître les opportunités d'entrer dans la vie active. Ce processus avait déjà été établi pour les sans-papiers régularisés (Adam et al., 2002 ; Marx et al., 2008).

La nationalité actuelle a aussi un impact significatif sur ce type de carrière quand nous considérons l'interaction entre nationalité belge et genre (cf. Tableau 5 en annexe). Les hommes qui ont acquis la nationalité belge ont une probabilité plus élevée d'avoir cette carrière progressivement active que ceux qui n'ont pas changé de nationalité. Pour les femmes, l'acquisition de la nationalité 
n'a aucun impact sur cette carrière. D'autres caractéristiques ont plus d'importance. La nationalité d'origine a un impact significatif sur le fait d'avoir ce type de carrière. Comparées aux personnes originaires d'Europe centrale, d'Europe de I'Est et du Caucase, les personnes originaires d'Afrique subsaharienne ont une probabilité plus élevée d'avoir une carrière progressivement active.

Le nombre d'années de procédure d'asile est également significatif. Mais contrairement à la carrière précédente, en comparaison d'une procédure d'asile de moins d'un an, les personnes qui ont une procédure d'asile de trois ans ou plus ont une probabilité plus faible d'avoir ce type de carrière. Autrement dit, les personnes arrivées en Belgique récemment, ont une probabilité supérieure d'avoir une carrière progressivement active par rapport aux personnes arrivées il y a plus de trois ans.

En regardant la situation sur le marché du travail, les personnes qui avaient déjà fait l'usage de la possibilité de travailler légalement avant l'obtention du statut de réfugié ont une plus faible probabilité d'avoir ce type de carrière que celles qui n'ont pas travaillé pendant leur procédure d'asile. Finalement, le contexte institutionnel ou économique n'a aucun impact significatif sur une insertion tardive sur le marché du travail.

\section{La carrière de dépendance sociale}

Cette carrière rassemble les 1432 personnes qui ne sont pas actives sur le marché du travail, que ce soit avant ou après l'obtention de statut. Les réfugiés qui la composent ont une carrière quasi entière de dépendance à l'aide sociale (cf. Figure 5). En moyenne, les réfugiés de cette carrière ont une aide sociale pendant $89 \%$ de la carrière observée. Ce type de carrière reprend $29 \%$ de la population étudiée. Cette carrière est, en un sens, complètement opposée à la première carrière.

Le tableau 3 en annexe montre les résultats de la troisième régression logistique multiple où la variable dépendante est le fait ou pas d'avoir une carrière de dépendance sociale. Les femmes ont une plus grande probabilité d'avoir une aide sociale pendant toute la carrière observée que les hommes. Concernant l'âge, nous voyons que les individus de dix-huit à vingt-cinq ans et de quarante-et-un à soixante-et-un ans ont une probabilité supérieure d'avoir ce type de carrière que ceux âgés de vingt-six à quarante ans. Cela signifie que la probabilité d'avoir une carrière de dépendance à l'aide sociale est curvilinéaire : la probabilité croit jusqu'à un certain âge puis décroît jusqu'à la retraite.

Encore une fois, les résultats démontrent que la situation familiale a un impact sur le fait d'avoir ce type de carrière, mais ce uniquement pour les femmes (cf. Tableau 5 en annexe). Les femmes célibataires avec enfant ont une probabilité plus grande d'avoir une aide sociale pendant toute leur carrière que les femmes en couple avec ou sans enfant et une probabilité moindre que les femmes célibataires sans enfant. L'impact de la situation familiale $n^{\prime}$ est pas significatif pour les hommes. Concernant l'origine nationale, les réfugiés originaires d'Europe de l'Est et du Caucase ont une probabilité plus grande d'avoir ce type de carrière lorsqu'ils sont comparés aux réfugiés venant d'Afrique subsaharienne. La nationalité actuelle a également un impact significatif sur ce type de carrière, mais à nouveau uniquement pour les hommes. Les hommes possédant la nationalité 
belge ont une probabilité plus faible d'avoir une carrière de dépendance à l'aide sociale que les individus n'ayant pas la nationalité belge.

\section{Figure 5 : La carrière de dépendance à l'aide sociale}

Distribution des statuts socio-économiques par trimestre (\%)

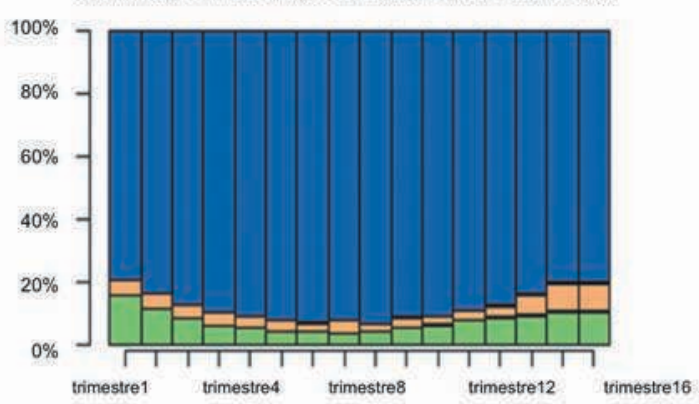

Dynamique longitudinale de la carrière (freq.)

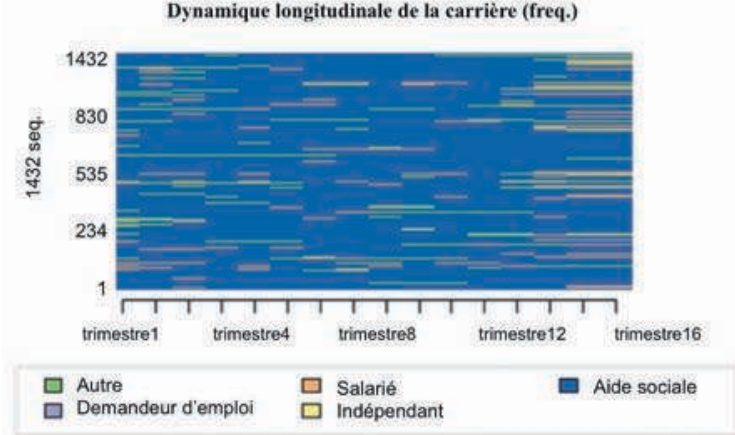

Le nombre d'années de la procédure d'asile est également significatif : plus le nombre d'années est faible, plus la probabilité d'avoir une carrière de dépendance sociale est grande. En regardant la situation sur le marché du travail, les personnes qui avaient déjà fait l'usage de la possibilité de travailler légalement avant l'obtention du statut de réfugié ont une plus faible probabilité de développer ce type de carrière que celles qui n'avaient pas travaillé pendant la procédure d'asile. Enfin, les résultats concernant l'impact des contextes institutionnels et économiques montrent que les réfugiés qui vivent en Wallonie au début de leur procédure de demande d'asile ont une probabilité significativement plus grande d'avoir une carrière de dépendance sociale que ceux qui vivent en Flandre. Les contextes institutionnels et économiques, entendus comme structures d'opportunités, particulièrement défavorables en Wallonie expliqueraient cette tendance.

\section{La carrière " autre "}

Le quatrième idéal-type est caractérisé par une carrière quasi unique du statut " autre " (cf. Figure 6). En moyenne, les 582 réfugiés de cette carrière ont ce statut pendant $82 \%$ de leur carrière étudiée. La grande majorité des personnes ayant ce statut $n$ 'ont aucun revenu légal et peuvent être considérées comme étant principalement des femmes/hommes au foyer. La figure 6 montre 
également que certaines personnes peuvent recourir à une aide sociale pendant une partie de leur carrière.

\section{Figure 6 : La carrière autre}

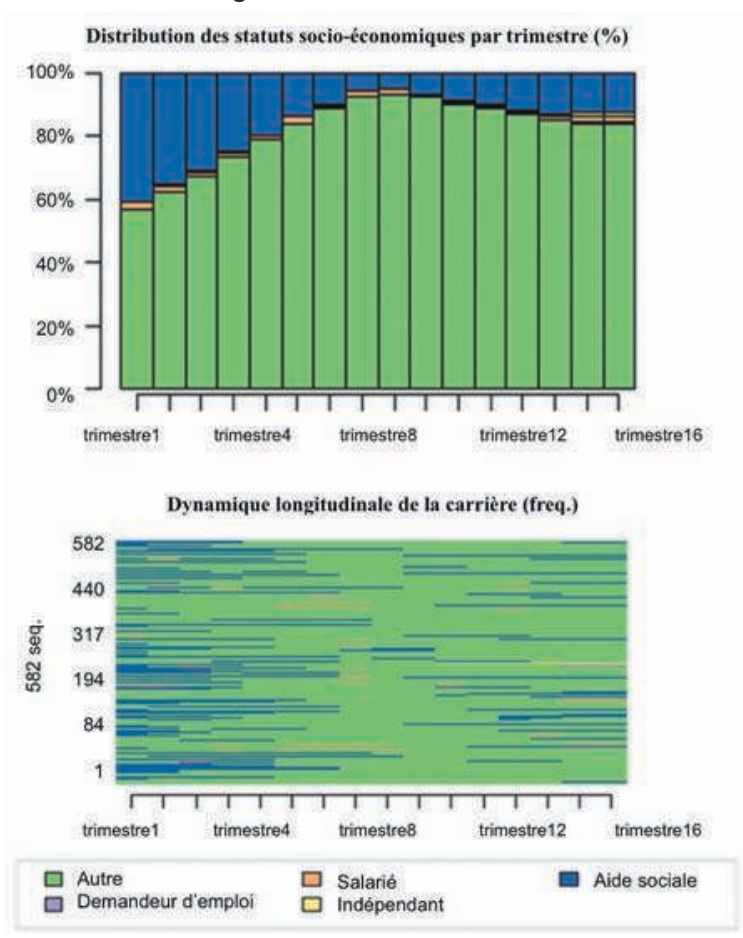

Le tableau 4 en annexe montre les résultats de la quatrième régression logistique multiple où la variable dépendante est le fait ou pas d'avoir une carrière avec le statut " autre ". Le genre a un impact significatif sur le fait d'avoir ce type de carrière. En effet, une femme a quatre fois plus de probabilité d'avoir une carrière avec un statut " autre " qu'un homme. La composition familiale joue également un rôle et contrairement aux autres types de carrière, la situation familiale des hommes au même titre que celle des femmes est significative (cf. Tableau 5 en annexe). Autrement dit, un homme ou une femme en couple avec ou sans enfant a une plus grande probabilité d'avoir une carrière " autre " qu'une personne célibataire avec ou sans enfant. Nous faisons I'hypothèse que l'absence d'un revenu régulier serait liée à la présence d'un partenaire qui apporterait les moyens de subsistance dans le couple.

Les résultats démontrent également que les réfugiés qui viennent d'un pays d'Europe centrale, d'Europe de l'Est et du Caucase ainsi que ceux venant du Moyen-Orient ont une probabilité plus élevée d'avoir ce type de carrière que ceux originaires d'Asie de l'Est et d'Afrique subsaharienne. Concernant la nationalité actuelle, avoir la nationalité belge a un impact significatif pour cette carrière, mais seulement pour les femmes. Les femmes ayant la nationalité belge ont une probabilité plus faible d'avoir une carrière autre que les personnes n'ayant pas la nationalité belge. 
En regardant la situation sur le marché du travail, les personnes ayant travaillé légalement avant l'obtention du statut de réfugié ont une plus faible probabilité $d^{\prime}$ avoir ce type de carrière que celles qui n'ont pas travaillé pendant la procédure d'asile. Ni le nombre d'années de la procédure d'asile, ni l'âge n'ont d'impact significatif sur le fait d'avoir une carrière sans revenu. Enfin, l'effet du contexte institutionnel et économique n'est significatif que pour Bruxelles.

\section{Les secteurs d'activités des réfugiés}

La stratification ethnique du marché du travail a été mise en lumière depuis de très nombreuses années (Piore, 1979) et elle a été réaffirmée au sujet du travail des réfugiés (Piché et al., 2002 ; Colic-Peisker et Tilbury, 2006). Si la dichotomie entre un secteur primaire et un secteur secondaire n'est plus aussi simple en raison de la fragmentation des statuts sur le marché du travail et en particulier du travail immigré (Rea, 2013), le changement de secteur d'activité à I'occasion du changement de statut juridique, en l'occurrence celui du passage de demandeur d'asile à celui de réfugié mérite d'être interrogé. C'est ce que nous avons fait avec les données qui étaient disponibles, et ce pour les deux premières carrières : carrière active et carrière progressivement active. Ceci a été possible par l'usage des codes NACE (Nomenclature statistique des Activités économiques) lorsqu'ils étaient enregistrés dans les données de la Banque carrefour de la sécurité sociale.

Concernant l'identification de la population, seuls 641 réfugiés ont un code NACE aux deux trimestres qui nous intéressent, à savoir le trimestre avant l'obtention du statut de réfugié et le dernier trimestre : 490 parmi la carrière active et 152 parmi la carrière progressivement active.

La figure 7 reprend les secteurs d'activité des réfugiés avant l'obtention de leur statut de réfugié et quatre ans après, et cela uniquement pour les personnes qui font partie des carrières actives et progressivement actives et qui ont eu un code NACE à la fois le trimestre avant l'obtention de leur statut et quatre ans après $(\mathrm{N}=642)$.

Le changement de statut conduit à un important changement de secteur d'activité pour ceux qui occupaient un emploi. Ceci est particulièrement vrai pour le secteur de l'agriculture, et plus exactement de I'horticulture. Avant I'obtention du statut de réfugié, un tiers d'entre eux travaille dans ce secteur, alors que seulement $2,5 \%$ y travaillent quatre ans après l'obtention de leur statut. Ce glissement de secteur s'est fait vers le secteur industriel, celui de l'administration publique, celui de la santé et du social ainsi que celui de l'hébergement, de la restauration et du commerce. Comme le démontre le monitoring socioéconomique produit pour l'ensemble des immigrés et de leurs descendants (SPF Emploi et le Centre interfédéral pour l'égalité des chances, 2015), les administrations publiques, et plus particulièrement les administrations locales constituent, un secteur qui est amené à absorber une partie des travailleurs qui sont discriminés sur le secteur privé.

Si on distingue les deux carrières, la différence est bien plus marquée pour la carrière progressivement active. Bien que les réfugiés développant une carrière progressivement active étaient peu nombreux à travailler pendant leur 
procédure d'asile, parmi ceux qui travaillaient, plus de deux tiers d'entre eux travaillaient dans le secteur de l'agriculture (68\%), alors qu'ils n'étaient plus que $2 \%$ quatre années après avoir été reconnus réfugiés. Depuis plus de trente ans, le secteur de l'horticulture constitue bien en Belgique une niche ethnique réservée aux immigrés, soit des demandeurs d'asile, soit des saisonniers.

Figure 7 : Secteurs d'activité avant l'obtention du statut de réfugié et quatre ans après (carrière active et carrière progressivement active) $(N=642)(e n \%)$

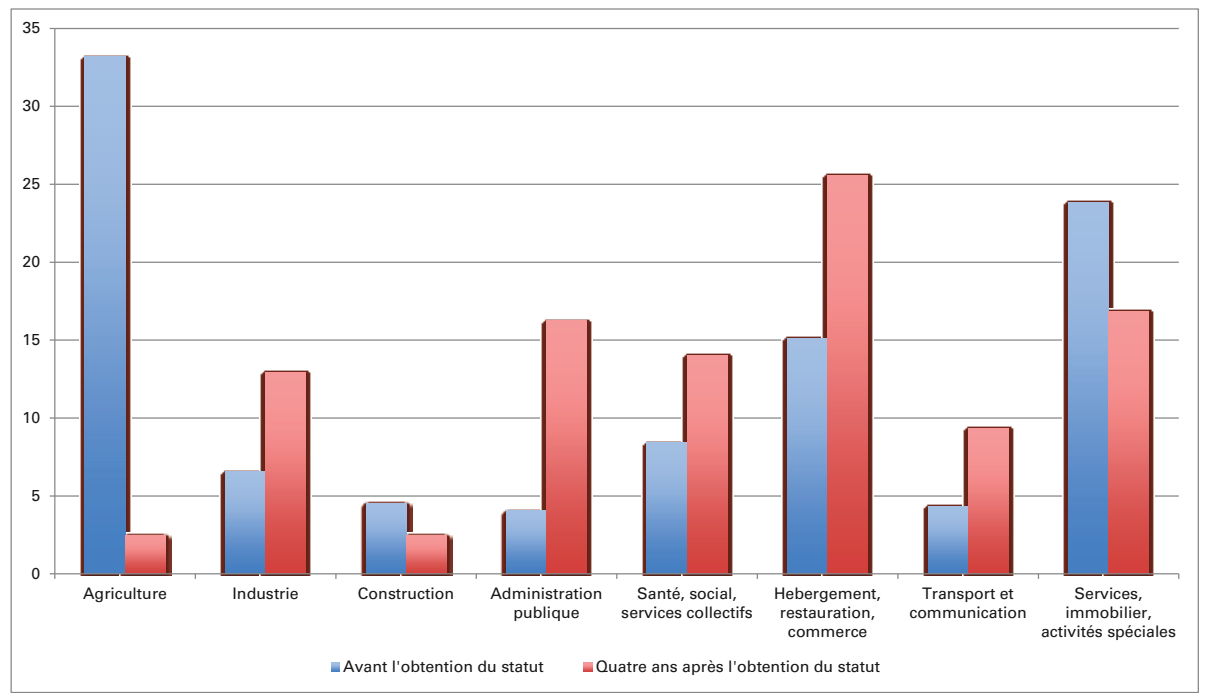

\section{Conclusion}

Partout en Europe, la réussite des immigrés sur le marché du travail tend à être inférieure à celle des citoyens natifs, et ce davantage en Belgique que dans les autres pays de l'OCDE (2008). Aucun déterminant unique ne semble être responsable de la situation. De nombreux facteurs interviennent dans le processus d'intégration du marché du travail et aucune mesure politique unique ne peut à elle seule offrir la clé du succès. La recherche effectuée révèle toutefois certains facteurs clés de l'intégration au marché du travail des réfugiés. Le temps joue un rôle crucial et contribue grandement au changement de la position socioéconomique des nouveaux migrants. Plus la durée de la présence du réfugié est longue, plus la probabilité que le réfugié a de trouver un travail et de cesser d'être dépendant de l'aide sociale est élevée. Disposer de plus de temps implique par ailleurs que les réfugiés peuvent mieux s'adapter à leur nouvel environnement, aux circonstances, aux institutions, à l'apprentissage de la langue nationale et au développement de réseaux sociaux. II faut du temps pour apprendre les normes et pratiques sociales concernant le pays d'installation. Le temps est également synonyme de possibilité d'apprendre la langue locale et de créer et développer un lien social.

La construction de quatre carrières d'insertion professionnelle met en évidence des formes variées, voire très contrastées. Un grand écart sépare la 
carrière active (34 \%) de la carrière de dépendance à l'aide sociale (29\%). Si l'on considère la période avant et après l'obtention du statut, certains réfugiés n'ont jamais été dépendants d'une aide sociale et ont presque toujours été actifs sur le marché du travail (carrière 1), tandis que d'autres ont été dépendants de l'aide sociale pratiquement sur toute la période observée (carrière 3). Si l'obtention du statut de réfugié constitue une manière de consolider une insertion professionnelle préalable pour la première carrière, il en va autrement pour la seconde. Pour la carrière progressivement active, l'obtention du statut de réfugié offre la possibilité d'accès à l'aide sociale. L'accès à l'aide sociale et les droits associés (formation, logement, etc.) devient un tremplin pour accéder à l'emploi. L'accès à l'aide sociale constitue ainsi un catalyseur de l'insertion.

Être présent sur le marché du travail à un stade précoce stimule la participation au marché du travail. Néanmoins, certains réfugiés sont plus vulnérables. Le genre, l'âge et la situation familiale sont des facteurs à prendre en compte. Les femmes rencontrent beaucoup plus de difficultés que les hommes, quel que soit leur statut familial. Les personnes qui vivent en famille s'en sortent manifestement mieux que les célibataires, mais les familles monoparentales (ayant majoritairement des femmes comme cheffe de famille) s'en sortent assurément plus mal. En particulier les mères célibataires. Elles dépendent plus des allocations d'aide sociale que les autres groupes et ont plus de difficultés à trouver la voie les menant vers le marché du travail. Par ailleurs, ce public spécifique ne bénéficie d'aucune politique ciblée de soutien. Par rapport aux réfugiés provenant des Balkans ou d'Asie centrale, les réfugiés originaires d'Afrique subsaharienne bénéficient d'un avantage en parlant une des langues nationales. La présence de longue date d'une communauté africaine en Belgique peut également contribuer à fournir une explication. Le réseau social peut s'avérer bénéfique dans la recherche d'un emploi. II convient toutefois de ne pas en surestimer l'importance. Le taux de chômage de la communauté africaine en Belgique est en général assez élevé.

Enfin, le contexte institutionnel et structurel joue un rôle très important dans les modalités d'intégration du marché du travail. La probabilité d'avoir un emploi en Flandre est le double de celle en Wallonie. La différence observée pour les réfugiés est toutefois plus forte que pour l'ensemble de la population. Le contexte institutionnel et structurel influence aussi les carrières des réfugiés reconnus comme cela a aussi été démontré pour les sans-papiers ayant bénéficié d'une régularisation de leur statut (Marx et al., 2008). Le contexte économique en Flandre avec un taux de chômage moins élevé que dans les deux autres Régions et une situation d'offres d'emploi plus favorable contribue à améliorer les chances $d$ 'insertion sur le marché du travail des réfugiés. Sans avoir d'éléments tangibles dans cette recherche, on peut supposer aussi que la politique d'intégration obligatoire en Flandre contribue d'une part, à améliorer les compétences linguistiques grâce aux cours de langue et d'autre part, le suivi institutionnel favorise aussi les contacts avec les institutions belges et flamandes favorisant ainsi le développement de liens faibles (Granovetter, 1973) utiles lors de la recherche d'emploi. Notons encore que l'influence du contexte est particulièrement importante pour les femmes réfugiées ce qui confirme leur position difficile sur le marché de l'emploi. 


\section{Références bibliographiques}

Abbott Andrew and Tsay Angela (2000) Sequence Analysis and Optimal Matching Methods in sociology. Review and Prospects, Sociological Methods \& Research, 29 (3), pp. 3-33.

Adam Ilke, Ben Mohamed Nadia, Kagné Bonaventure, Martiniello Marco et Rea Andrea (2002) Histoires sans-papiers, Bruxelles, Édition Vista, 227 p.

Banque nationale de Belgique (2011) Rapport 2011, Bruxelles, BNB, 111 p.

Bevelander Pieter (2011) The employment integration of resettled refugees, asylum claimants, and family reunion migrants in Sweden, Refugee Survey Quarterly, 30 (1), pp. 22-43.

Bloch Alice (2007) Refugees in the UK labour market: The conflict between economic integration and policy-led labour market restriction, Journal of Social Policy, 37 (1), pp. 21-36.

Burggraeve Koen et Piton Céline (2016) Les conséquences économiques de I'afflux de réfugiés en Belgique, Revue économique, juin, pp. 49-67.

Colic-Peisker Veljka and Tilbury Farida (2006) Employment niches for recent refugees: Segmented labour market in twenty-first century Australia, Journal of Refugee Studies, 19 (2), pp. 203-229.

DeMaris Alfred (1992) Logit Modeling: Practical Applications, Newbury Park, CA, Sage, $92 \mathrm{p}$.

Granovetter Mark (1973) The Strength of Weak Ties, American Journal of Sociology, 78 (6), pp. 1360-1380.

Halpin Brendan and Chan Tak Wing (1998) Class careers as sequences: An optimal matching analysis of work-life histories, European Sociological Review, 14 (2), pp. 111-130.

Hauff Edvard and Vaglum Per (1993) Integration of Vietnamese refugees into the Norwegian labour market: the impact of war trauma, International Migration Review, 17 (2), pp. 388-405.

Hjarno Jan (1991) Migrants and refugees on the Danish labour market, New Community, 18 (1), pp. 75-87.

Korac Maja (2003) Integration and How We Facilitate It: A comparative Study of the Settlement Experiences of Refugees in Italy and the Netherlands, Sociology, 37 (1), pp. 51-68.

Krahn Harvey, Derwing Tracey, Mulder Marlene and Wilkinson Lori (2000) Educated and Underemployed: Refugee Integration into Canadian Labour Market, Journal of International Migration and Integration, 1 (1), pp. 59-84.

Lamba Navjot (2003) The Employment Experience of Canadian Refugees: Measuring the Impact of Human and Social Capital on Quality of Employment, Revue Canadienne de Sociologie, 40 (1), pp. 45-64.

Martiniello Marco and Rea Andrea (2014) The concept of migratory careers: Elements for a new theoretical perspective of contemporary human mobility, Current Sociology, 62 (7), pp. 1079-1096.

Martiniello Marco, Rea Andrea, Timmerman Christiane et Wets Johan (Éds.) (2010) Nouvelles migrations et nouveaux migrants en Belgique, Gent, Academia Press, 339 p. 
Marx Ive, Rea Andrea, Verbist Gerlinde, Godin Marie et Corluy Vincent (2008) La situation sociale et économique des personnes ayant bénéficié de la procédure de régularisation en 2000 (loi du 22 décembre 1999), Bruxelles, Centre pour I'égalité des chances et la lutte contre le racisme, 175 p., [en ligne]. URL : http:// germe.ulb.ac.be/uploads/pdf/articles\%20online/anciens/BeforeAfter-rapport.pdf

Montgomery Randall (1991) Predicting Vietnamese refugee adjustment to western Canada, International Migration Review, 29 (1), pp. 89-111.

Mussche Ninke, Corluy Vincent and Marx Ive (2010) Satisfying Labour Demand through Migration in Belgium, Brussels, European Migration Network, 96 p.

OCDE (2016) Making Integration Work: Refugees and Others in Need of Protection, Paris, OECD Publishing, $74 \mathrm{p}$.

OCDE (2008) Jobs for Immigrants, vol. 2: Labour Market Integration in Belgium, France, the Netherlands and Portugal, Paris, OECD Publishing, 334 p.

Piché Victor, Renaud Jean et Gingras Lucie (2002) L'insertion économique des nouveaux immigrants dans le marché du travail à Montréal : une approche longitudinale, Population, 57 (1), pp. 63-89.

Piguet Étienne et Wimmer Andreas (2000) Les nouveaux Gastarbeiter ? Les réfugiés sur le marché du travail Suisse, Journal of International Migration and Integration, 1 (2), pp. 233-257.

Piore Michael (1979) Birds of Passage: Migrant Labor and Industrial Societies, Cambridge, Cambridge University Press, 229 p.

Rea Andrea (2013) Les nouvelles figures du travailleur immigré : fragmentation des statuts d'emploi et européanisation des migrations, Revue Européenne des Migrations Internationales, 29 (2), pp. 15-35.

Rea Andrea and Wets Johan (Eds.) (2015) Long and Winding Road to Employment. The migratory carrers of refugees in Belgium, Gand, Academia Press, 171 p., [online]. URL: http://www.myria.be/files/Careers_LR.pdf

Renaud Jean, Piché Victor and Godin Jean-François (2003) “One's bad and the other is worse": Differences in economic integration between asylum seekers and refugees selected abroad, Canadian Ethnic Studies, 35 (2), pp. 86-99.

Robette Nicolas (2011) Explorer et décrire les parcours de vie: les typologies de trajectoires, Paris, CEPED, $86 \mathrm{p}$.

SPF Emploi et le Centre interfédéral pour l'égalité des chances (2015) Monitoring socio-économique 2015 : marché du travail et origine, 265 p., [en ligne]. URL : http://www.emploi.belgique.be/publicationDefault.aspx?id=44125

Timmerman Christiane, Martiniello Marco, Rea Andrea and Wets Johan (Eds.) (2014) New Dynamics in Female Migration and Integration, London, Routledge, $258 \mathrm{p}$.

Tran Thanh (1991) Sponsorship and employment status among Indochinese refugees in the United States, International Migration Review, 25 (3), pp. 536-550.

Wets Johan (Ed.) (2009) Realisatie van onderzoeksmogelijkheden naar ulterieure trajecten en tendensen by ex-aanvragers van een regularisatie, ROUTE project (BELSPO), Leuven, HIVA-Katholieke Universiteit, 215 p.

Wooden Mark (1991) The Experience of Refugees in the Australian Labour Market, International Migration Review, 25 (3), pp. 514-535.

Yu Soojin, Ouellet Estelle and Warmington Aangelyn (2007) Refugee integration in Canada: A survey of empirical evidence and existing service, Refuge, 2 (24), pp. 17-34. 


\section{Annexes}

Tableau 2 : Description des variables indépendantes utilisées dans les analyses

\begin{tabular}{|c|c|}
\hline Variables indépendantes & Description \\
\hline \multirow{3}{*}{ Genre } & Variable binaire \\
\hline & Femme - catégorie de référence \\
\hline & Homme \\
\hline \multirow{4}{*}{ Âge } & $\begin{array}{l}\text { Âge lors de la reconnaissance du statut de réfugié } \\
\text { (le début de la période observée) }\end{array}$ \\
\hline & Entre 18 et 25 ans \\
\hline & Entre 26 et 40 ans - catégorie de référence \\
\hline & Entre 41 et 61 ans \\
\hline \multirow{7}{*}{ Situation familiale } & Variable catégorielle \\
\hline & Célibataire - catégorie de référence \\
\hline & Couple \\
\hline & Parents \\
\hline & Parent célibataire \\
\hline & Jeune \\
\hline & Autre situation familiale \\
\hline \multirow{7}{*}{ Nationalité d'origine } & Variable catégorielle \\
\hline & Afrique subsaharienne - catégorie de référence \\
\hline & Europe centrale \\
\hline & Europe de l'Est et Caucase \\
\hline & Moyen-Orient \\
\hline & Asie de l'Est \\
\hline & Autres pays \\
\hline \multirow[b]{2}{*}{ Nationalité belge } & Variable binaire \\
\hline & $\begin{array}{l}\text { Avoir la nationalité belge à n'importe quel moment de la } \\
\text { période étudiée }\end{array}$ \\
\hline \multirow{4}{*}{$\begin{array}{l}\text { Durée de la procédure de demande } \\
\text { d'asile = durée du séjour } \\
\text { en Belgique }\end{array}$} & Variable catégorielle \\
\hline & Un an ou moins - catégorie de référence \\
\hline & Plus d'un an et moins de trois ans \\
\hline & Trois ans ou plus \\
\hline \multirow{2}{*}{$\begin{array}{l}\text { Participation au marché de l'emploi } \\
\text { lors du trimestre précédent } \\
\text { l'obtention du statut de réfugié }\end{array}$} & Variable binaire \\
\hline & $\begin{array}{l}\text { Employé, indépendant ou demandeur d'emploi indemnisé } \\
\text { lors du trimestre précédent l'obtention du statut de réfugié }\end{array}$ \\
\hline \multirow{4}{*}{ Région } & Première région de résidence en Belgique \\
\hline & Wallonie - catégorie de référence \\
\hline & Bruxelles \\
\hline & Flandre \\
\hline
\end{tabular}


Tableau 3 : Fréquence des variables indépendantes par carrière et par genre

\begin{tabular}{|c|c|c|c|c|c|c|c|c|c|c|}
\hline \multirow[b]{2}{*}{ Homme ou Femme } & \multicolumn{2}{|c|}{ Carrière 1} & \multicolumn{2}{|c|}{ Carrière 2} & \multicolumn{2}{|c|}{ Carrière 3} & \multicolumn{2}{|c|}{ Carrière 4} & \multicolumn{2}{|c|}{ Total } \\
\hline & $\mathrm{H}$ & $\mathrm{F}$ & $\mathrm{H}$ & $\mathrm{F}$ & $\mathrm{H}$ & $\mathrm{F}$ & $\mathrm{H}$ & $\mathrm{F}$ & $\mathrm{H}$ & $\mathrm{F}$ \\
\hline \multicolumn{11}{|l|}{ Âge } \\
\hline $18-25$ & 230 & 109 & 201 & 142 & 193 & 251 & 29 & 100 & 653 & 602 \\
\hline $26-40$ & 684 & 354 & 457 & 197 & 292 & 343 & 74 & 239 & 1507 & 1133 \\
\hline $41-61$ & 171 & 108 & 119 & 83 & 159 & 194 & 51 & 89 & 500 & 474 \\
\hline \multicolumn{11}{|l|}{ Situation familiale } \\
\hline Célibataire & 676 & 184 & 504 & 189 & 369 & 272 & 54 & 55 & 1603 & 700 \\
\hline Couple & 35 & 29 & 20 & 10 & 18 & 23 & 12 & 22 & 85 & 84 \\
\hline Parents & 249 & 240 & 166 & 38 & 175 & 90 & 60 & 272 & 650 & 640 \\
\hline Parent célibataire & 20 & 84 & 20 & 147 & 13 & 317 & 2 & 24 & 55 & 572 \\
\hline Jeune & 13 & 5 & 17 & 8 & 21 & 25 & 7 & 8 & 58 & 46 \\
\hline Autre situation familiale & 89 & 27 & 47 & 30 & 45 & 59 & 10 & 40 & 191 & 156 \\
\hline Données manquantes & 3 & 2 & 3 & 0 & 3 & 2 & 9 & 7 & 18 & 11 \\
\hline \multicolumn{11}{|l|}{ Nationalité d'origine } \\
\hline Afrique subsaharienne & 520 & 254 & 298 & 246 & 211 & 325 & 49 & 85 & 1078 & 910 \\
\hline Europe centrale & 56 & 33 & 21 & 15 & 35 & 29 & 8 & 41 & 120 & 118 \\
\hline Europe de I'Est et Caucase & 316 & 215 & 296 & 120 & 305 & 356 & 72 & 240 & 989 & 931 \\
\hline Moyen-Orient & 84 & 29 & 87 & 16 & 55 & 44 & 18 & 50 & 244 & 139 \\
\hline Asie de l'Est & 72 & 23 & 47 & 19 & 17 & 15 & 1 & 1 & 137 & 58 \\
\hline Autres pays & 10 & 9 & 5 & 1 & 4 & 4 & 1 & 0 & 20 & 14 \\
\hline Données manquantes & 27 & 8 & 23 & 5 & 17 & 15 & 5 & 11 & 72 & 39 \\
\hline \multicolumn{11}{|l|}{ Nationalité belge } \\
\hline Avoir la nationalité belge & 622 & 388 & 409 & 255 & 293 & 457 & 73 & 230 & 1397 & 1330 \\
\hline Ne pas avoir la nationalité belge & 463 & 183 & 368 & 167 & 351 & 331 & 81 & 198 & 1269 & 879 \\
\hline \multicolumn{11}{|c|}{ Durée de la procédure d'asile $=$ durée du séjour en Belgique } \\
\hline Un an ou moins & 206 & 107 & 261 & 114 & 232 & 246 & 32 & 140 & 731 & 607 \\
\hline Plus d'un an et moins de trois ans & 332 & 158 & 247 & 151 & 189 & 250 & 52 & 135 & 820 & 694 \\
\hline Trois ans ou plus & 547 & 306 & 269 & 157 & 223 & 292 & 70 & 153 & 1109 & 908 \\
\hline \multicolumn{11}{|c|}{ Participation au marché de l'emploi lors du trimestre précédent l'obtention du statut de réfugié } \\
\hline N’a pas participé & 690 & 444 & 678 & 395 & 597 & 765 & 143 & 421 & 2108 & 2025 \\
\hline A participé & 395 & 127 & 99 & 27 & 47 & 23 & 11 & 7 & 552 & 184 \\
\hline \multicolumn{11}{|l|}{ Région } \\
\hline Wallonie & 220 & 117 & 150 & 94 & 152 & 186 & 36 & 105 & 558 & 502 \\
\hline Bruxelles & 626 & 299 & 454 & 234 & 358 & 464 & 84 & 210 & 1522 & 1207 \\
\hline Flandre & 235 & 148 & 170 & 93 & 130 & 131 & 31 & 110 & 566 & 482 \\
\hline Données manquantes & 4 & 7 & 3 & 1 & 4 & 7 & 3 & 3 & 14 & 18 \\
\hline Total & 1085 & 571 & 777 & 422 & 644 & 788 & 154 & 428 & 2660 & 2209 \\
\hline
\end{tabular}


Tableau 4 : Régressions logistiques (odds ratio) pour les quatre carrières

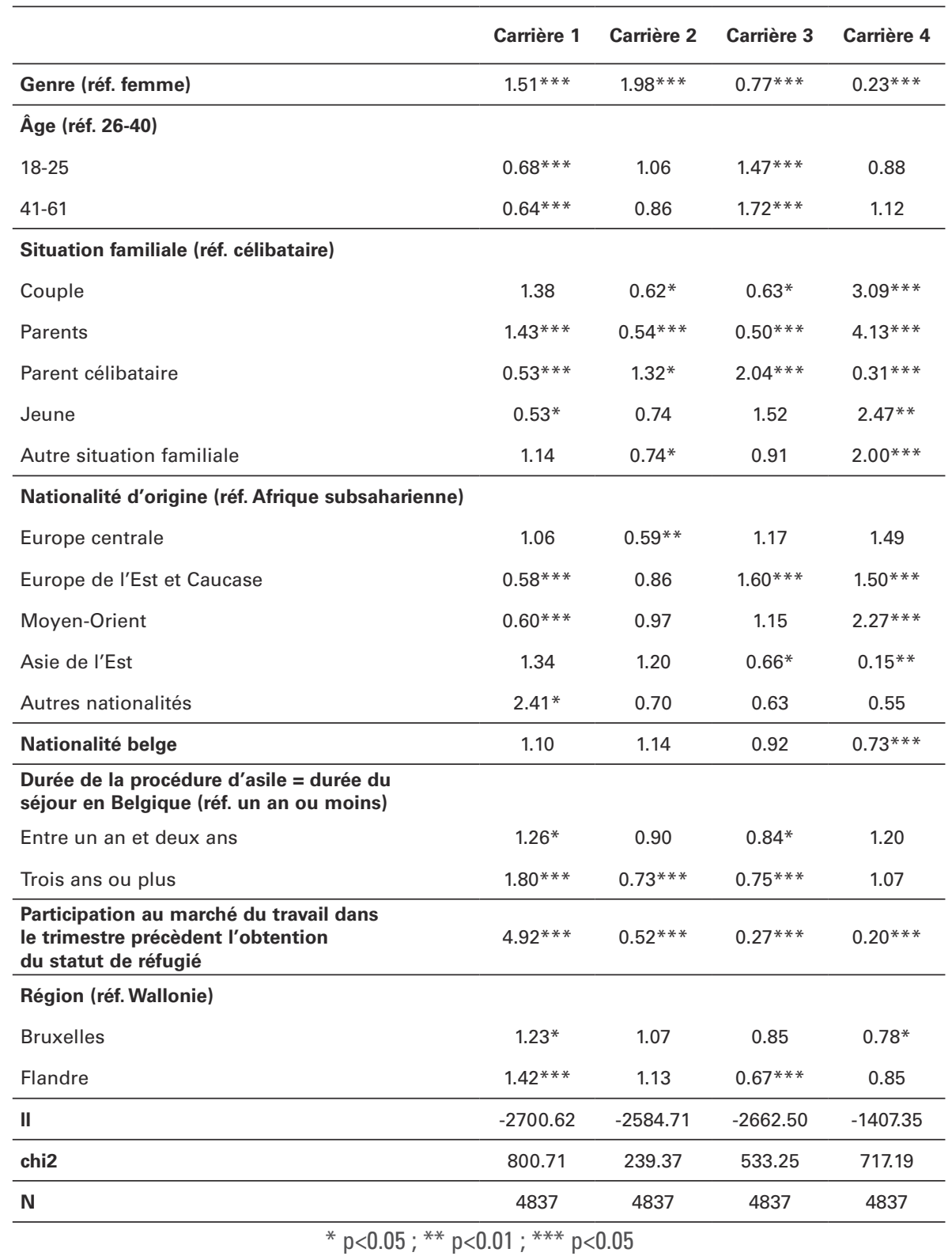

Note : Le total $(\mathrm{N})$ n'équivaut pas à 4869 à cause des données manquantes des variables " situation familiale ", " nationalité " et " région ". 
Tableau 5 : Régressions logistiques (odds ratio) par carrière et par genre

\begin{tabular}{|c|c|c|c|c|c|c|c|c|}
\hline & \multicolumn{2}{|c|}{ Cluster 1} & \multicolumn{2}{|c|}{ Cluster 2} & \multicolumn{2}{|c|}{ Cluster 3} & \multicolumn{2}{|c|}{ Cluster 4} \\
\hline & Homme & Femme & Homme & Femme & Homme & Femme & Homme & Femme \\
\hline \multicolumn{9}{|l|}{ Âge (réf. 26-40) } \\
\hline $18-25$ & $0.74 * *$ & $0.57 * * *$ & 0.95 & 1.26 & $1.57 * * *$ & $1.44^{* *}$ & 0.92 & 0.83 \\
\hline $41-61$ & $0.64 * * *$ & $0.71 *$ & $0.72 * *$ & 1.04 & $1.93 * * *$ & $1.37^{*}$ & $1.75^{* *}$ & 0.94 \\
\hline \multicolumn{9}{|c|}{ Situation familiale (réf. célibataire) } \\
\hline Couple & 1.13 & $1.89 *$ & 0.69 & $0.48^{*}$ & 0.73 & $0.48^{* *}$ & $3.75^{* * *}$ & $3.04 * * *$ \\
\hline Parents & 1.05 & $2.24 * * *$ & 0.80 & $0.24 * * *$ & 0.97 & $0.20 * * *$ & $2.06 * *$ & $6.03^{* * *}$ \\
\hline Parent célibataire & 1.02 & $0.55^{* * *}$ & 1.32 & 1.15 & 0.77 & $1.71 * * *$ & 0.66 & $0.38 * * *$ \\
\hline Jeune & 0.60 & 0.47 & 0.76 & 0.64 & 1.33 & 1.45 & $3.83^{* *}$ & 2.03 \\
\hline $\begin{array}{l}\text { Autre situation } \\
\text { familiale }\end{array}$ & $1.50^{*}$ & 0.76 & $0.70^{*}$ & 0.77 & 0.87 & 0.82 & 1.14 & $2.80 * * *$ \\
\hline \multicolumn{9}{|c|}{ Nationalité d'origine (réf. Afrique subsaharienne) } \\
\hline Europe centrale & 1.33 & 0.77 & $0.53^{*}$ & 0.73 & 1.35 & 1.05 & 0.98 & $1.79 *$ \\
\hline $\begin{array}{l}\text { Europe de l'Est } \\
\text { et Caucase }\end{array}$ & $0.56^{* * *}$ & $0.57 * * *$ & 1.14 & $0.58 * * *$ & $1.63 * * *$ & $1.74 * * *$ & 1.15 & $1.72 * * *$ \\
\hline Moyen-Orient & $0.64 * *$ & $0.49 * *$ & 1.34 & $0.46^{* *}$ & 1.10 & 1.37 & 1.35 & $3.14 * * *$ \\
\hline Asie de l'Est & 1.40 & 1.16 & 1.17 & 1.55 & $0.55^{*}$ & 0.91 & 0.18 & $0.12^{*}$ \\
\hline Autres nationalités & 2.01 & 2.76 & 0.69 & 0.85 & 0.68 & 0.63 & 0.85 & 0.38 \\
\hline Nationalité belge & 1.06 & 1.18 & $1.22^{*}$ & 1.04 & $0.81 *$ & 1.01 & 0.71 & $0.75^{*}$ \\
\hline \multicolumn{9}{|c|}{ Durée de la procédure d'asile = durée du séjour en Belgique (réf. un an ou moins) } \\
\hline $\begin{array}{l}\text { Entre un an } \\
\text { et deux ans }\end{array}$ & $1.35^{*}$ & 1.12 & 0.82 & 1.11 & 0.81 & 0.87 & $1.77^{*}$ & 0.99 \\
\hline Trois ans ou plus & $1.83 * * *$ & $1.71 * * *$ & $0.64 * * *$ & 0.93 & $0.70 * *$ & 0.78 & $1.82^{*}$ & 0.80 \\
\hline $\begin{array}{l}\text { Participation au } \\
\text { marché du travail } \\
\text { dans le trimestre } \\
\text { précèdent } \\
\text { l'obtention du } \\
\text { statut de réfugié }\end{array}$ & $4.42 * * *$ & $6.71 * * *$ & $0.49 * * *$ & 0.70 & $0.28 * * *$ & $0.26 * * *$ & $0.25^{* * *}$ & $0.14^{* * *}$ \\
\hline \multicolumn{9}{|c|}{ Région (réf. Wallonie) } \\
\hline Bruxelles & 1.26 & $1.68^{* *}$ & 1.10 & 1.26 & $0.74^{*}$ & $0.60 * * *$ & 0.81 & 0.82 \\
\hline Flandre & 1.16 & $1.34^{*}$ & 1.10 & 1.06 & $0.79 *$ & 0.93 & 0.88 & $0.70^{*}$ \\
\hline II & -1593.69 & -1078.18 & -1549.74 & -986.67 & -1359.68 & -1246.57 & -534.19 & -846.27 \\
\hline chi2 & 391.72 & 342.85 & 98.96 & 170.88 & 208.34 & 361.08 & 89.61 & 463.11 \\
\hline $\mathrm{N}$ & 2646 & 2191 & 2646 & 2191 & 2646 & 2191 & 2646 & 2191 \\
\hline
\end{tabular}

Note : Le total $(\mathrm{N})$ n'équivaut pas à 4869 à cause des données manquantes des variables " situation familiale ", " nationalité " et « région ". 


\section{Barbara Herman et Andrea Rea}

\section{La longue et sinueuse route vers l'emploi : les carrières d'insertion professionnelle des réfugiés sur le marché du travail en Belgique}

Partout en Europe, l'insertion des immigrés sur le marché du travail tend à être inférieure à celle des citoyens natifs et ce, davantage en Belgique que dans les autres pays de I'OCDE (2009). Aucun déterminant unique ne semble être responsable de la situation. Cet article révèle toutefois certains facteurs clés de I'intégration au marché du travail des réfugiés. Les résultats, obtenus à partir de données administratives longitudinales en Belgique, mettent en évidence que le temps joue un rôle crucial et contribue grandement au changement de la position socioéconomique des nouveaux migrants. Plus la durée de la présence du réfugié est longue, plus la probabilité que le réfugié a de trouver un travail et de cesser d'être dépendant de l'aide sociale est élevée. Aussi, grâce à l'identification de carrières types d'insertion professionnelle, obtenue par le biais d'analyse séquentielle, les résultats démontrent qu'être présent sur le marché du travail à un stade précoce stimule la participation ultérieure à celui-ci. Néanmoins, certains réfugiés restent plus vulnérables. Le genre, l'âge et la situation familiale sont des facteurs à prendre en compte. Enfin, le contexte institutionnel et structurel joue un rôle très important dans les modalités d'intégration du marché du travail.

\section{The Long and Winding Road to Employment: Labour Market Careers of Refugees in Belgium}

All over Europe, immigrants' labour market outcomes tend to lag behind those of the native-born and in Belgium even farther than in other OECD countries (2009). No single aspect seems to be responsible of this phenomenon. This article however reveals some key factors in the labour market integration of refugees. The results were obtained from longitudinal administrative data in Belgium and show that time plays a crucial role, greatly contributing to change new immigrants' socio-economic position. The longer a person is present in the country, the more likely this person would find a job and cease dependence on social welfare. Moreover, the results show that being present on the labour market in an early stage stimulates further participation within the labour market. Results were obtained through sequential analysis that built the identification of socioeconomic integration paths. Some groups remain more however vulnerable. Gender, age and family situation are factors to be taken into account. Finally, the institutional context plays a very important role for labour market integration. 


\section{La larga y tortuosa vía hasta el empleo: carreras de inserción profesional de refugiados en el mercado de trabajo en Bélgica}

En toda la Europa, la inserción de inmigrantes en el mercado de trabajo tiende a ser inferior a la de los ciudadanos nativos, y sobre todo en Bélgica en comparación con otros países de la OCDE (2009). Ningún determinante único parece ser responsable de esta situación. Todavía, esta contribución revela algunos de los factores llave de la integración de refugiados en el mercado de trabajo. Los resultados, obtenidos de datos administrativos longitudinales en Bélgica, evidencian que el tiempo desempeña un papel crucial y contribuye de forma significativa para cambiar la posición socioeconómica de los nuevos inmigrantes. Cuánto más larga es la duración de la presencia del refugiado en Bélgica, más elevada es la probabilidad que encuentre un trabajo y que deje de depender de una ayuda social. Además, los resultados de la identificación de carreras profesionales típicas, construidas a partir de un análisis secuencial, demuestran que estar presente en el mercado de trabajo a un estado precoz estimula una participación futura. Sin embargo, algunos refugiados siguen más vulnerables. El género, la edad y la situación familiar son factores que se han de tener en cuenta. Por último, el contexto institucional y estructural desempeña una función muy importante en la modalidades de integración en el mercado de trabajo. 